SMALL INTESTINE

\title{
Cellular detection of sst2A receptors in human gastrointestinal tissue
}

\author{
M Gugger, B Waser, A Kappeler, A Schonbrunn, J C Reubi
}

Gut 2004;53:1431-1436. doi: 10.1136/gut.2004.042002

See end of article for authors' affiliations

.....................

Correspondence to: Professor J C Reubi, Division of Cell Biology and Experimental Cancer Research, Institute of Pathology, University of Berne, PO Box 62 Murtenstrasse $31, \mathrm{CH}-$ 3010 Berne, Switzerland; reubi@pathology.unibe.ch

Revised version received 24 March 2004

Accepted for publication 30 March 2004

\begin{abstract}
Background and aim: Many neuroendocrine gastrointestinal tumours express receptors for the regulatory peptide somatostatin. Among the five existing somatostatin receptor (sst) subtypes, sst2A is the most frequently expressed in these tumours. However, little information is available about the cellular location of sst2A in corresponding non-neoplastic epithelial tissues.

Methods: We searched for sst2A immunoreactive cells in non-neoplastic gastrointestinal tissues, and evaluated their number and immunohistochemical characteristics with neuroendocrine markers.

Results: The gastric antrum showed numerous sst2A cells, situated in the epithelium, corresponding to gastrin containing neuroendocrine cells, while the gastric corpus was largely devoid of sst2A cells, including enterochromaffin-like cells. The remaining foregut, namely the duodenum and proximal jejunum, also contained a large number of sst2A cells, all being neuroendocrine cells and many of them characterised as gastrin cells. Sst2A cells were also detected in the midgut, in low numbers in the epithelium of the distal jejunum and ileum, but not in the appendix vermiformis, the caecum, or the hindgut, despite the large number of neuroendocrine cells present in this area. In addition, sst2A cells were found in the whole gastrointestinal tract in the myenteric and submucosal plexus.

Conclusions: While sst2A receptors on antral gastrin cells presumably mediate somatostatin inhibition of gastrin secretion, the effects of somatostatin on motility and ion transport in the lower gastrointestinal tract may be mediated by sst2A receptors in the neural plexus. These data provide a molecular basis for the physiological actions of somatostatin in human gastrointestinal tissue.
\end{abstract}

S omatostatin receptors (sst) are frequently expressed in neuroendocrine tumours of the gut ${ }^{12}$ and hence provide the basis for in vivo targeting of these tumours with somatostatin analogues. ${ }^{3-5}$ For instance, the beneficial effect of octreotide therapy, a stable analogue of somatostatin, on hormonal secretion in somatostatin receptor expressing neuroendocrine tumours of the gut $^{3}$ is well documented. Furthermore, $\left[{ }^{111}\right.$ In-DTPA $\left.{ }^{0}\right]$-octreotide has been successfully used to detect these tumours by receptor scintigraphy in vivo. ${ }^{4}$ Moreover, radiotherapy with ${ }^{90}$ Y-DOTATOC has been performed in clinical phase I studies showing remission or stabilisation of disease in the majority of patients with end stage cancer. ${ }^{4}$

Compared with cancer, information on the cellular localisation of the sst protein in non-neoplastic human gut tissues is limited. In vitro receptor autoradiography detected sst in the non-neoplastic gut mucosa as well as in lymphoid tissue, nerve plexus, circular smooth muscle at the mucosa directed margin, and the vasculature ${ }^{26-8}$ but without precise cellular localisation and without receptor subtype specification. In situ mRNA hybridisation and reverse transcriptionpolymerase chain reaction in non-neoplastic colon tissue detected all subtypes, most often sst 2 or sst5, localised in the epithelium as well as in the lamina propria. ${ }^{9}$

In the present study, we took advantage of a very well characterised sst antibody, R2-88, ${ }^{11}$ that identifies one of the most abundant sst subtypes in neuroendocrine tumours, namely the splice variant sst2A. ${ }^{12}$ Immunohistochemistry can be done on formalin fixed tissue, the most often available tissue fixation, and single cells are detectable. Hence we have evaluated the presence of sst2A protein in non-neoplastic formalin fixed tissue of various parts of the human gut. We compared the results with neuroendocrine markers on serial sections. In the gastric antrum, sst receptor autoradiographic results on frozen tissue were correlated with those of sst $2 \mathrm{~A}$ immunohistochemistry. Knowledge of the distribution of sst2 in normal human tissues provides new insights into the physiology of somatostatin. It may also be of value in understanding the carcinogenesis of sst 2 positive neuroendocrine tumours.

\section{MATERIAL AND METHODS}

\section{Tissues}

Formalin fixed paraffin embedded tissue samples from resection specimens collected at the Institute of Pathology were selected, showing non-neoplastic tissue with a normal histology on sections cut perpendicular to the mucosal surface. Tissue samples were collected per anatomical location (table 1).

\section{Receptor autoradiography}

Frozen sections of the gastric antrum and corpus were incubated for two hours at room temperature with ${ }^{125} \mathrm{I}$ labelled $\left[\mathrm{Tyr}^{3}\right]$-octreotide, identifying preferentially sst2, or with ${ }^{125}$ I- $\left[\right.$ Leu $^{8}$, DTrp ${ }^{22}$, Tyr $\left.{ }^{25}\right]$-SS-28 ( ${ }^{125}$ I-LTT-SS-28) as universal ligand, as described previously. ${ }^{1}$ Displacement of these ligands from their binding sites was evaluated with sst selective analogues, ${ }^{2}$ namely the sst 2 selective L-779,976, the sst3 selective 315-164-15 (also referred to as sst3-ODN-8), and the sst5 selective L-817,818 in studies with ${ }^{125}$ I-labelled $\left[\mathrm{Tyr}^{3}\right]$-octreotide, and additionally the sstl selective $\mathrm{CH}-288$ and the sst4 selective L-803,087 in studies with ${ }^{125}$ I-LTT-SS28. After sections were washed, they were apposed to BiomaxMR films (Kodak, Lausanne, Switzerland) and exposed for one week in $x$ ray cassettes. Non-specific binding was determined in parallel sections incubated with the same

Abbreviations: sst, somatostatin receptor; $E C L$, enterochromaffin-like; TBS, Tris buffered saline; CgA, chromograninA; HDC, histidine decarboxylase 


\section{Table 1 Tissue selection}

\begin{tabular}{ll}
\hline Location & No of samples \\
\hline Gastric corpus & 4 \\
$\quad$ Antrum & 5 \\
Duodenum & 4 \\
Jejunum proximal & 2 \\
Distal & 3 \\
lleum & 5 \\
Appendix vermiformis & 3 \\
Colon & 3 \\
Rectum & 3 \\
\hline
\end{tabular}

Total number of patients, 27

In two patients, two tissue samples with a different location were investigated and three tissue samples contained two locations. Specimens contained a malignant neoplasm in 21 patients and inflammatory lesions in six patients.

concentration of labelled peptide in the presence of $10^{-6} \mathrm{~mol} / \mathrm{l}$ of unlabeled octreotide or SS-28, respectively.

\section{Immunohistochemistry}

We used the polyclonal rabbit antibody $\mathrm{R} 2-88$ raised against a unique sequence in the carboxyl terminal region of the sst $2 \mathrm{~A}$ receptor, corresponding to amino acids 339-359 in the rat protein. The identical sequence is found in human, rat, and mouse sst $2 \mathrm{~A}$ receptor proteins, and as a result the antibody was shown to be highly specific on human tissue, both fresh frozen and formalin fixed paraffin embedded samples. ${ }^{12}$ Formalin fixed paraffin embedded sections, cut at $2-3 \mu \mathrm{m}$, were dewaxed, rehydrated, and boiled in $100 \mathrm{mM}$ Tris with $5 \%$ urea, $\mathrm{pH} 9.5$, in a pressure cooker for five minutes. After pretreatment (and following all subsequent steps), sections were washed in Tris buffered saline (TBS). Sections were incubated overnight at room temperature with the sst $2 \mathrm{~A}$ antibody diluted 1:2000 in TBS containing 1\% bovine serum albumin, 5\% normal goat serum, and $0.1 \% \mathrm{NaN}_{3}$. Sections were then incubated with a biotinylated goat-antirabbit antibody (1:200; same buffer as for the primary antibody; DakoCytomation, Glostrup, Denmark) and thereafter with avidin-biotin complex/horseradish peroxidase (DakoCytomation; 1:120 in TBS). Finally, sst2A stained sections were developed in $0.05 \%$ 3,3'-diaminobenzidine and $\mathrm{H}_{2} \mathrm{O}_{2}$, weakly counterstained with haematoxylin, and mounted. For all cases, an additional adjacent tissue section was incubated with the primary sst $2 \mathrm{~A}$ antibody that had been preabsorbed with an excess (100 nM) of the peptide antigen prior to staining, to serve as a specificity control.

In addition, we performed immunohistochemical staining on serial sections with the following commercially available rabbit polyclonal antibodies: gastrin (1:300), chromograninA $(\mathrm{CgA} ; 1: 300)$, protein $\mathrm{S} 100$ (1:800, as a marker of peripheral nerve cells) (all from DakoCytomation), and histidine decarboxylase (HDC; 1:2000; Progen, Heidelberg, Germany). After dewaxing and rehydrating, sections were boiled in $10 \mathrm{mM}$ citrate buffer, $\mathrm{pH}$ 6.0, for five minutes (CgA, gastrin) or 10 minutes (HDC) in a pressure cooker. After pretreatment, sections were incubated for 60 minutes at room temperature with CgA, protein S100, HDC, or gastrin diluted in TBS containing $0.5 \%$ casein sodium salt and $5 \%$ goat serum. Subsequently, sections were incubated with biotinylated secondary antibody (1:500) and thereafter with streptavidin-biotin complex/alkaline phosphatase (1:200 in TBS) (both DakoCytomation). Sections were developed with new fuchsin-naphtol AS-BI, weakly counterstained with haematoxylin, and mounted.

In selected specimens from the antrum, we performed immunofluorescence double staining on formalin fixed paraffin embedded sections. The primary antibodies included the above polyclonal rabbit antibody sst2A and a monoclonal mouse-antihuman CgA antibody (Clone DAK-A3; DakoCytomation). Sections were cut at $2-3 \mu \mathrm{m}$, dewaxed, rehydrated, and boiled in $100 \mathrm{mM}$ Tris with 5\% urea, $\mathrm{pH}$ 9.5, in a microwave oven. Sections were incubated overnight at room temperature with CgA diluted 1:100 and sst2A diluted $1: 1000$ in TBS containing $0.5 \%$ casein sodium salt and $5 \%$ goat serum. Red (Alexa Fluor 546) and green (Alexa Fluor 488) fluorochrome labelled secondary antibodies (Molecular Probes, Eugene, Oregon, USA) were used to visualise target structures. Goat-antirabbit Ig antibodies were used at 1:600 and goat-antimouse Ig at 1:300, and were incubated for 45 minutes.

\section{Estimation of receptor positive cells/cm mucosa}

Tissue samples were photographed at low magnification $(1.25 \times$ objective, eyepiece $10 \times)$. The length of the lamina muscularis was then measured on photographed sections that were cut perpendicular to the mucosal surface. On serial sections, the number of immunohistochemically positive cells was counted in the epithelium, at a medium magnification $(10 \times$ objective, eyepiece $10 \times)$, permitting visualisation of the entire mucosal height in the visual field. For various investigated anatomical locations, tissue samples contained a total mucosal length of a minimum of $3.3 \mathrm{~cm}$ (corpus) and up to a maximum of $7.2 \mathrm{~cm}$ mucosa (colon). Median (SD) count/cm mucosa in each anatomical location was calculated. Count/cm mucosa could then be compared with published counts $/ \mathrm{cm}^{2}$ based on a section thickness of $2 \mu \mathrm{m}$.

\section{RESULTS}

Sst2A receptor proteins were expressed immunohistochemically at several locations in the non-neoplastic gut (table 2).

In the upper gut, sst2A cells were most numerous (table 2) in the mucosa of the gastric antrum, situated in the

Table 2 Sst2A expressing cells in the gut epithelium

\begin{tabular}{lllll}
\hline Location & $\mathbf{n}$ & $\begin{array}{l}\text { No of sst2A } \\
\text { expressing cells/ } \\
\mathbf{c m}^{*}\end{array}$ & $\begin{array}{l}\text { No of gastrin expressing } \\
\text { cells in corresponding } \\
\text { region* }\end{array}$ & $\begin{array}{l}\text { No of CgA expressing cells in } \\
\text { corresponding region* }\end{array}$ \\
\hline Gastric corpus & 4 & 0 & 0 & $1150(436)$ \\
Gastric antrum & 5 & $449(243)$ & $918(134)$ & $1364(248)$ \\
Duodenum & 4 & $275(101)$ & $472(147)$ & $1365(205)$ \\
Jejunum proximal & 2 & $261(41)$ & $298(65)$ & $1034(38)$ \\
Distal & 3 & $27(33)$ & $44(7)$ & $373(25)$ \\
lleum & 5 & $13(13)$ & $70(56)$ & $406(87)$ \\
Appendix vermiformis & 3 & 0 & $N D$ & $123(38)$ \\
Colon & 3 & 0 & $N D$ & $580(44)$ \\
Rectum & 3 & 0 & ND & \\
\hline n, number of tissue samples per location. & & \\
*Number of cells (SD)/cm of mucosa. & \\
CgA, chromograninA; ND, not done. &
\end{tabular}




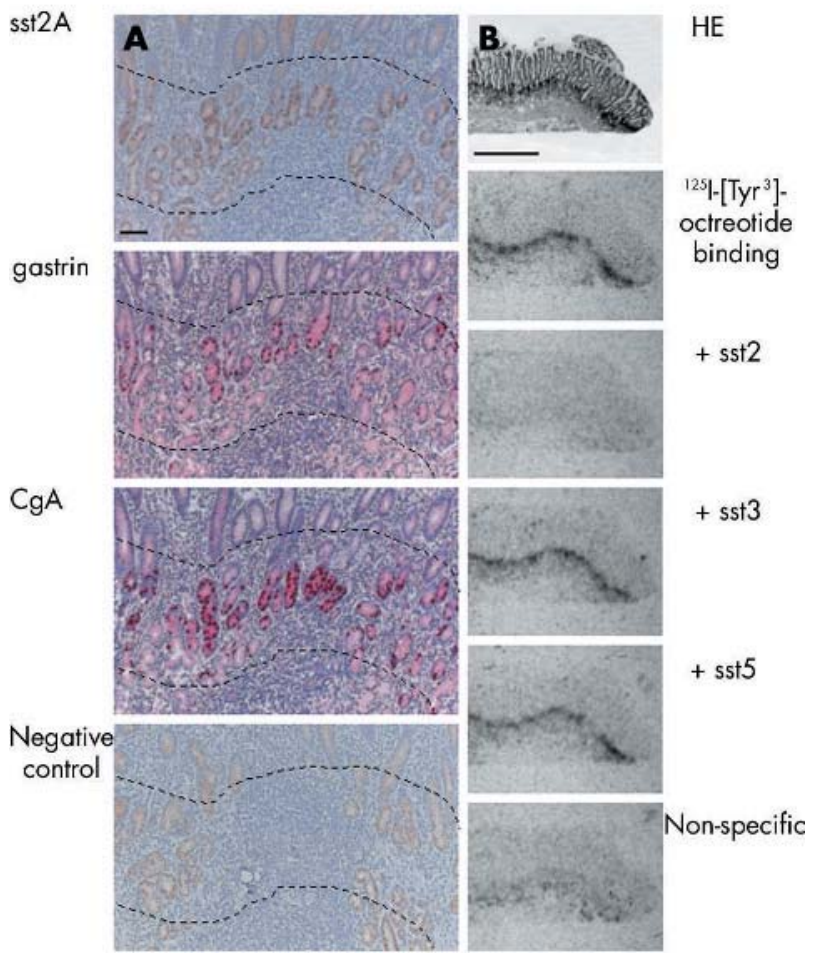

Figure 1 Comparison of immunohistochemistry (A) and receptor autoradiography (B) in the gastric antrum. (A) Localisation of immunohistochemically detected cells in the gastric antrum. 1st row: somatostatin receptor $2 \mathrm{~A}$ (sst2A) cells lie at the neck of the glands. They represent a linear zone, indicated between the broken lines. Staining of the more superficial layers was non-membranous and non-specific. 2nd and 3rd rows: Gastrin and $\mathrm{CgA}$ cells, respectively, were localised in the same region as sst2A cells. 4 th row: Peptide preabsorption as a negative control for sst2A showing residual non-specific non-membranous staining. Bar $100 \mu \mathrm{m}$. (B) Receptor autoradiographic illustration of sst receptor subtypes in the gastric antrum. 1st row: Haematoxylin and eosin (HE) stained section. 2nd row: Autoradiogram showing total binding of the ligand ${ }^{125}$-[Tyr3]-octreotide at the neck of the glands. 3rd row: Autoradiogram showing ${ }^{125}$-[Tyr3]-octreotide binding in the presence of $100 \mathrm{nM}$ of an sst2 selective ligand $(L-779,976)$. Complete displacement of the radioligand is seen. 4th and 5th rows:

Autoradiograms showing ${ }^{125}$-[Tyr3]-octreotide binding in the presence of $100 \mathrm{nM}$ of an sst3 selective ligand (315-164-15) and $100 \mathrm{nM}$ of an sst5 selective ligand $(L-817,818)$. No displacement of binding was seen. 6th row: Non-specific binding (in the presence of $100 \mathrm{nM}$ octreotide). Complete displacement of labelling by the sst2 selective ligand indicates the presence of sst2 receptors. Bar $1000 \mu \mathrm{m}$.

epithelium at the transition from the foveolae to the glands. At low power magnification, sst2A positive cells formed a linear zone comparable with CgA and gastrin positive areas (fig 1A). This correlated well with the linear zone of binding at the same location in the receptor autoradiography experiments with ${ }^{125}$ I-labelled $\left[\mathrm{Tyr}^{3}\right]$-octreotide (fig $\mathrm{IB}$ ). Displacement of the ligand with unlabelled sst2, sst3, and sst5 selective analogues revealed the predominance of subtype 2 (fig 1B). At high power magnification, sst2A were exquisitely membrane bound in basket shaped cells located at the basement membrane in the mucosal foveolae (fig 2). On serial sections, these cells all belonged to a larger population of CgA cells in this area. In addition, they were always positive for gastrin located in the cytoplasm (figs 1, 2).

Results of serial sections were further confirmed by double immunofluorescence staining with a combination of sst $2 \mathrm{~A}$ and CgA, revealing individual double positive cells in the foveolae at the neck of the glands, basket or oval shaped, and basement membrane based, as described above, with
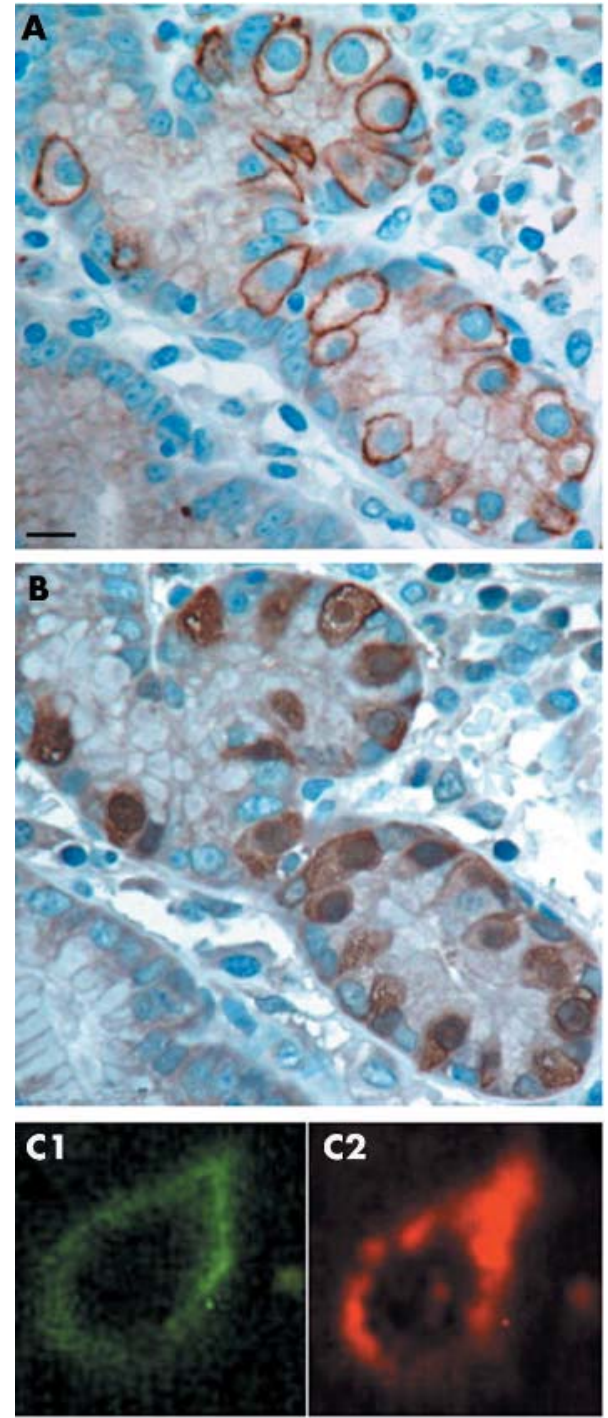

Figure 2 Immunohistochemical characterisation of somatostatin receptor 2A (sst2A) cells in the antrum on serial tissue sections and by fluorescence labelled double staining. Immunohistochemistry on serial sections. (A) Specific and very strong membranous staining of sst2A protein in basket shaped cells in the neck of the gland. (B) Sst2A cells in (A) were identified as gastrin cells by cytoplasmic staining for gastrin protein in the same cells. Bar $10 \mu \mathrm{m}$. Double staining by immunofluorescence for sst2A (C1, Alexa Fluor488) and chromograninA $(\mathrm{CgA})(\mathrm{C} 2$, Alexa Fluor546). Membranous sst2A and cytoplasmic $\mathrm{CgA}$ staining were detected in the same cell.

membrane bound sst2A and cytoplasmic CgA (fig 2). We detected no sst $2 \mathrm{~A}$ cells in the epithelium of the gastric corpus. On the one hand, we identified in this location all neuroendocrine cells, as measured by CgA immunohistochemistry. On the other hand, we detected enterochromaffinlike (ECL) cells by immunostaining of specific HDC. Theses ECL cells were present in the lower part of the corpus. Even when focusing on these ECL cells in serial sections, no sst2A were detected. In agreement with this, we did not detect sst 2 in the mucosa of the corpus on receptor autoradiography. Interestingly, in samples containing both normal mucosa as well as hyperplastic ECL cell lesions and/or ECL cell tumours, only hyperplasia and tumours expressed large amounts of membrane bound sst2A (see below).

The duodenum and proximal jejunum also contained epithelial sst $2 \mathrm{~A}$ cells while only rare sst $2 \mathrm{~A}$ cells were detected in the distal jejunum and ileum (table 2). Figure 3 shows a 


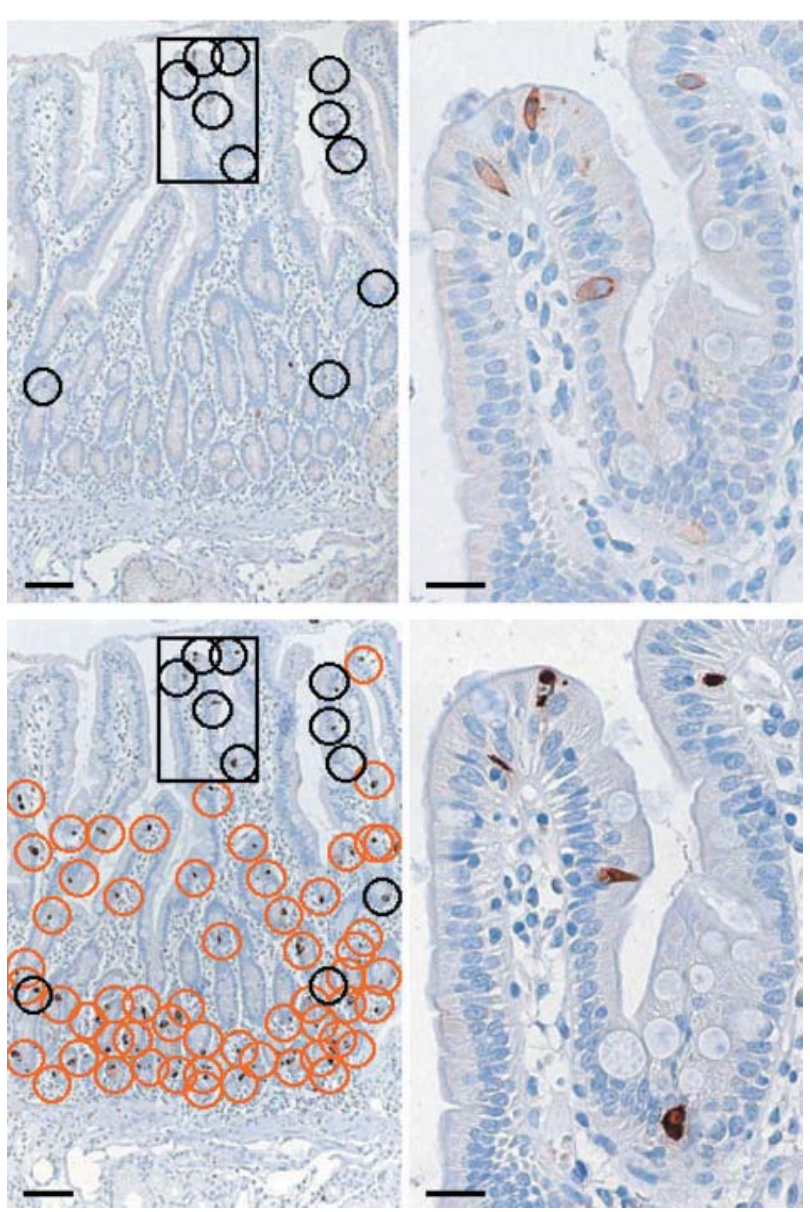

Figure 3 Representative data for somatostatin receptor 2A (sst2A) and chromogranin $\mathrm{A}(\mathrm{CgA})$ immunohistochemistry on serial sections of the duodenum. Bars $100 \mu \mathrm{m}$. Insets on the right represent enlarged views of the framed areas (bars $25 \mu \mathrm{m}$ ). First row: Sst2A immunohistochemistry; second row: $\mathrm{CgA}$ immunohistochemistry. Stained epithelial cells were encircled black or orange. Black circles denote individual sst2A cells that were also stained for $\mathrm{CgA}$ on the serial section in the second row. In this example, all sst2A cells were identified as $\mathrm{CgA}$ positive. For comparison, orange circles identified $\mathrm{CgA}$ positive cells that were not sst2A positive. Sst2A cells were heterogeneously distributed and often situated in villi. Tall and flask-like cells revealed mainly membranous sst2A and cytoplasmic $\mathrm{CgA}$ staining.

representative tissue section from the duodenum with sst $2 \mathrm{~A}$ and CgA immunohistochemical staining on serial sections. Stained epithelial cells were identified as black or orange circles. Black circles denote individual sst2A positive cells that were also positive for $\mathrm{CgA}$ on the serial section. In this example, all sst $2 \mathrm{~A}$ cells were identified as CgA cells. The orange circles identified CgA positive cells that were not sst2A positive, giving an idea of the quantitative ratio between the different cell types in this location. Note that sst2A cells were heterogeneously distributed and often situated in villi.

In the appendix vermiformis, colon and rectum, no sst $2 \mathrm{~A}$ cells were detected in the epithelium, despite large number of detected neuroendocrine CgA positive cells (table 2). Conversely, in the submucosa and tunica muscularis, ganglionic cells were strikingly sst2A positive (fig 4) and identified on serial sections as neural cells by their positivity for the peripheral nerve cell marker protein S100. We found sst2A cells in the myenteric and submucosal plexus in the whole gastrointestinal tract, including the stomach, duodenum, jejunum, ileum, appendix vermiformis, colon, and rectum.

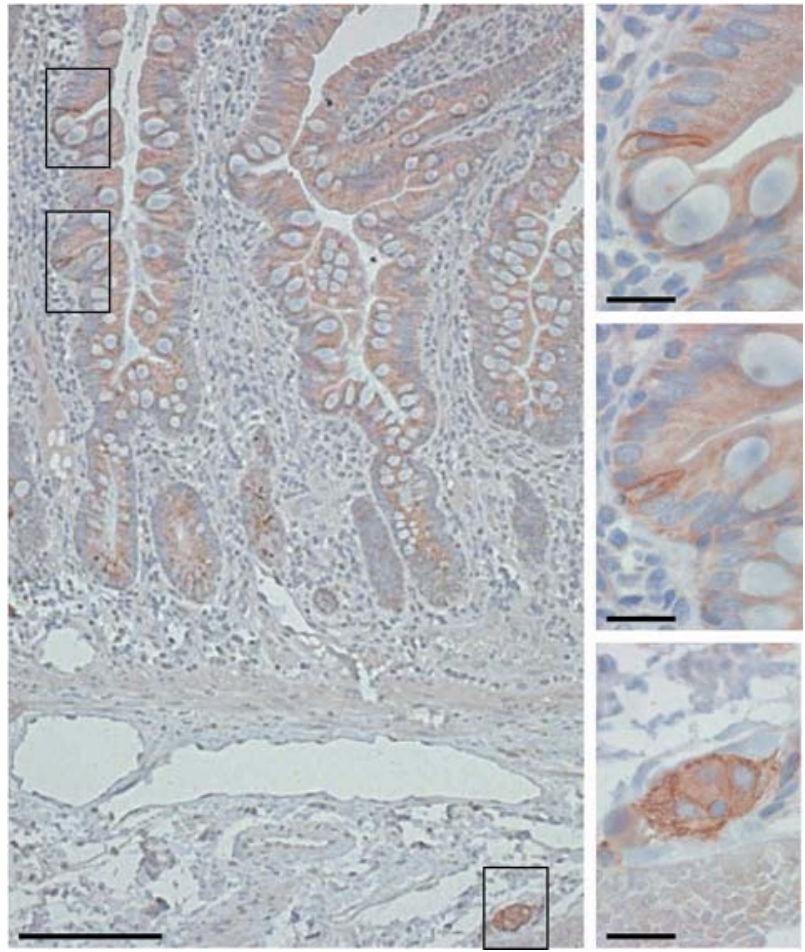

Figure 4 Representative somatostatin receptor $2 \mathrm{~A}$ (sst2A) immunohistochemistry of the ileum. Sst2A cells were often clustered (framed areas at the top left). Bar $100 \mu \mathrm{m}$. Insets on the right represent enlarged views of the three framed areas (bars $25 \mu \mathrm{m})$. Top and middle: Epithelial, tall, and flask-shaped sst2A cells, showing membranous staining. Bottom: Ganglionic cells revealed membranous and/or faint cytoplasmic reactivity.

It should be noted that the number of cells/cm mucosa detected by $\mathrm{CgA}$ and gastrin immunohistochemistry in the present study were in the range of previous investigations. ${ }^{13} 14$

For comparison, fig 5 shows the extent of sst2A positivity in various gut neuroendocrine lesions, using identical immunohistochemical methodology: ECL cell hyperplasia as well as an ECL cell tumour of the stomach, gastrinoma of the duodenum, and an ileal carcinoid, all strongly expressing sst2A at the membrane of hyperplastic or neoplastic cells.

\section{DISCUSSION}

This is the first description of immunohistochemically detected sst2A protein at the cellular level in the epithelium of non-neoplastic human gut. There were large differences in the amounts of sst2A cells in different areas of the gut. Sst $2 \mathrm{~A}$ cells correspond to defined cell populations but they do not necessarily parallel the neuroendocrine cell population. In gut epithelium, sst2A positive cells are neuroendocrine cells. In the gastric antrum, sst2A cells represent mostly neuroendocrine gastrin cells whereas in the lower gut neuroendocrine epithelial cells do not express sst2A. However, sst2A receptors are clearly expressed in the submucosal and myenteric plexus throughout the whole of the gastrointestinal tract, a finding that extends our previous immunohistochemical observation of sst2A cells in the neural plexus of the human colon. ${ }^{7}$

The sst2A antibody R2-88 has previously been well characterised and shown to be highly specific for the sst $2 \mathrm{~A}$ receptor ${ }^{11}$ in animal and human tissues and to correlate well with other methodologies such as in vitro sst autoradiography or mRNA in situ hybridisation. ${ }^{12}$ The present study further emphasises the excellent characteristics of the antibody by showing: good correlation between in vitro receptor autoradiography and immunohistochemistry in the gastric 

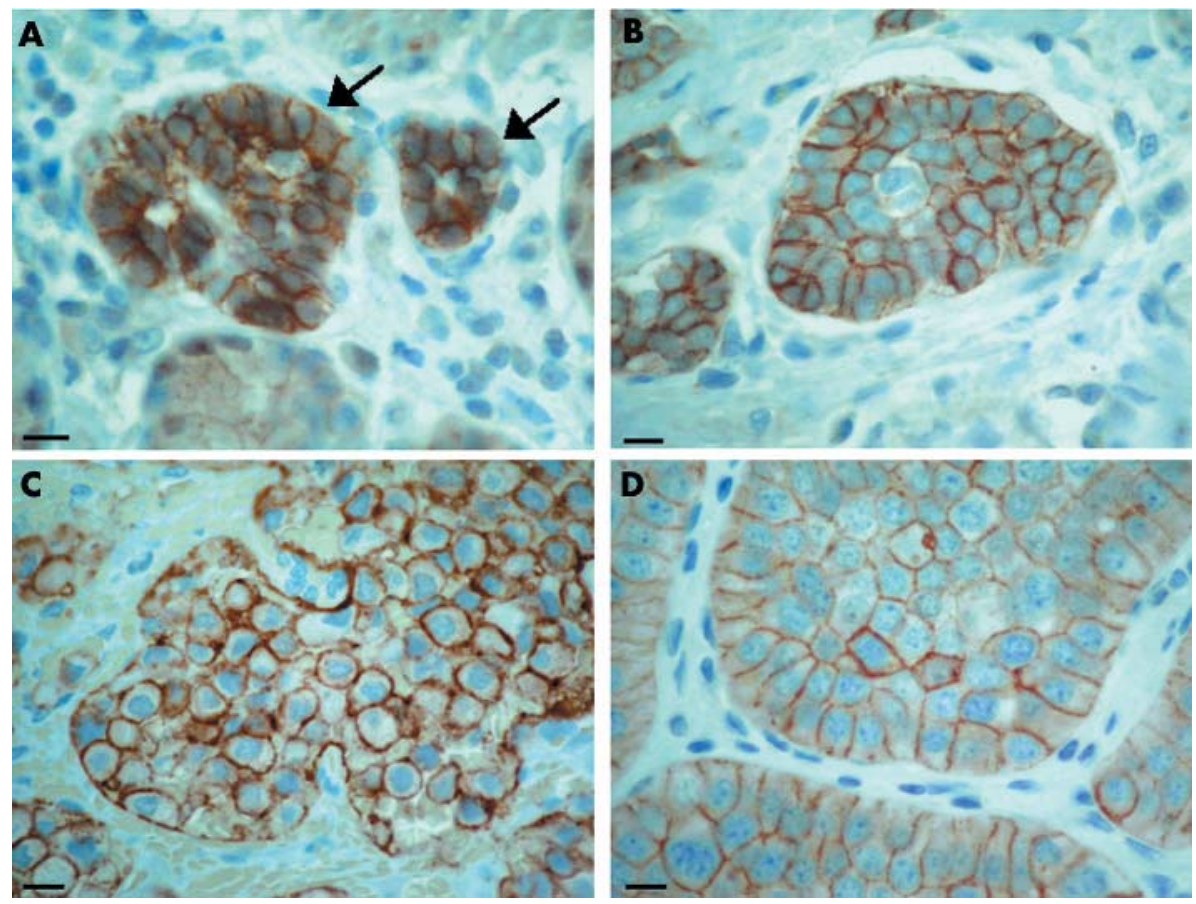

Figure 5 Immunohistochemical somatostatin receptor 2A (sst2A) positive neuroendocrine lesions of the gut. Membrane bound sst2A staining was seen in hyperplastic enterochromaffin-like (ECL) cell aggregates (arrows, A) and in an $E C L$ cell tumour of the gastric corpus (B), as well as in a gastrinoma (C) of the Papilla vateri and in a carcinoid (D) of the jejunum. Bars $10 \mu \mathrm{m}$.

antrum where sst2A cells were dense and numerous enough to generate an autoradiographic signal; consistent results in the normal part of surgical samples of patients with different malignant neoplasms and different inflammatory lesions; and unambiguous characterisation of sst $2 \mathrm{~A}$ cells by other immunohistochemical markers.

Detection of sst2A cells in the epithelium in the foregut and in the neural plexus throughout the gastrointestinal tract adds to our understanding of the cellular mechanisms involved in the physiological actions of somatostatin in humans. Although substantial information is available regarding the action of somatostatin in the gastrointestinal tract in mice and rats, ${ }^{15-21}$ direct investigation using human tissues is necessary to extrapolate from animals to humans as species differences are widely observed with peptides and peptide receptors. ${ }^{5}$ In the human foregut (stomach to proximal jejunum), the sst2A receptor protein on antral gastrin cells is likely to represent the molecular basis for the inhibitory effect of somatostatin on gastrin secretion, ${ }^{22}$ mediated in a paracrine way through cytoplasmic processes of somatostatin cells projecting onto gastrin cells. ${ }^{23}$ In the rodent, there is as yet no evidence for sst2A expression in antral gastrin cells. ${ }^{21}$ Conversely, there is good evidence in the rodent for a multiple somatostatin action on parietal cells and ECL cells through sst2A receptors ${ }^{17} 2021$ (Reubi unpublished data) whereas our present human data do not suggest a somatostatin action through sst2A in cells of the corpus. An action of somatostatin in the corpus of the human stomach may however be mediated by sstl, as suggested recently by in vitro receptor autoradiographic data. ${ }^{2}$ The present human data clearly favour a predominant role of somatostatin through sst $2 \mathrm{~A}$ at the level of gastrin cells: the high number of sst2A expressing gastrin cells and the consistency of the labelling throughout the different tissue samples may underline the importance of this mechanism in human tissue. The present data therefore clearly indicate a species difference in the location of sst $2 \mathrm{~A}$ receptors in the stomach between humans and rodents. In the duodenum and proximal jejunum, sst2A cells were found to always represent neuroendocrine cells, subtyped most often as gastrin cells. In the midgut (distal jejunum to caecum) and hindgut (colon and rectum), where rare and no sst2A cells, respectively, were found in the epithelium, the action of somatostatin may be primarily mediated by sst $2 \mathrm{~A}$ receptors on neural cells in the plexus submucosus and myentericus. These receptors, found throughout the neural plexus of the gastrointestinal tract, may represent the molecular basis for sst2 mediated inhibition of gut motility by somatostatin. ${ }^{24-26}$ Those in the plexus submucosus may also represent the molecular basis for somatostatin inhibition of colonic ion secretion, as shown in a human in vitro mode ${ }^{27}$ of colonic tissue samples stripped of underlining connective tissue. The submucosal plexus is the only location in the mucosa and submucosa of the colon where we detected sst $2 \mathrm{~A}$ cells. In the rodent, sst $2 \mathrm{~A}$ positive cells are also found in this location. ${ }^{20}$

Can the present study add to our understanding of the carcinogenesis of gut neuroendocrine tumours? While specific areas of the human gut have a large number of sst $2 \mathrm{~A}$ receptor containing cells, our data show that other areas, such as the gastric corpus as well as the mid and hindgut, have no or surprisingly few sst2 expressing cells in nonneoplastic epithelium. Interestingly, mid and hindgut are regions where the majority of gut neuroendocrine tumours ${ }^{28}$ arise, and these are characterised by high sst2 receptor expression. On the basis of the sst 2 expressing cells identified in the mid and hindgut in the present study, it is therefore difficult to define the cell of origin of mid and hindgut neuroendocrine tumours. ${ }^{14}{ }^{29-34}$ In the gastric corpus however, from which neuroendocrine tumours arise that are predominantly ECL cell tumours with high sst 2 expression, ${ }^{35}$ one may speculate that the absence of sst2A in normal ECL cells (even in those patients with sst2A positive tumours) indicates that ECL cell tumours and preinvasive hyperplastic lesions may express sst2 de novo. 


\section{Authors' affiliations}

M Gugger, B Waser, A Kappeler, J C Reubi, Division of Cell Biology and Experimental Cancer Research, Institute of Pathology, University of Bern, Switzerland

A Schonbrunn, Department of Integrative Biology and Pharmacology, University of Texas Health Sciences Center Houston, Houston, Texas, USA

\section{REFERENCES}

1 Reubi JC, Kvols LK, Waser B, et al. Detection of somatostatin receptors in surgical and percutaneous needle biopsy samples of carcinoids and islet cell carcinomas. Cancer Res 1990;50:5969-77.

2 Reubi JC, Waser B, Schaer JC, et al. Somatostatin receptor sst1-sst5 expression in normal and neoplastic human tissues using receptor autoradiography with subtype-selective ligands. Eur J Nucl Med $2001 ; 28: 836-46$

3 Lamberts SW, van der Lely AJ, de Herder WW, et al. Octreotide. N Engl J Med 1996;334:246-54.

4 Kwekkeboom D, Krenning EP, de Jong M. Peptide receptor imaging and therapy. J Nucl Med 2000;41:1704-13.

5 Reubi JC. Peptide receptors as molecular targets for cancer diagnosis and therapy. Endocr Rev 2003;24:389-427.

6 Reubi JC, Horisberger U, Waser B, et al. Preferential location of somatostatin receptors in germinal centers of human gut lymphoid tissue. Gastroenterology 1992; 103:1207-14

7 Reubi JC, Laissue JA, Waser B, et al. Immunohistochemical detection of somatostatin sst2a receptors in the lymphatic, smooth muscular, and peripheral nervous systems of the human gastrointestinal tract: facts and artifacts. J Clin Endocrinol Metab 1999;84:2942-50.

8 Rettenbacher M, Reubi JC. Localization and characterization of neuropeptide receptors in human colon. Naunyn Schmiedebergs Arch Pharmacol 2001;364:291-304.

9 Vuaroqueaux V, Dutour A, Bourhim N, et al. Increased expression of the mRNA encoding the somatostatin receptor subtype five in human colorecta adenocarcinoma. J Mol Endocrinol 2000;24:397-408.

10 Casini Raggi C, Calabro A, Renzi D, et al. Quantitative evaluation of somatostatin receptor subtype 2 expression in sporadic colorectal tumor and in the corresponding normal mucosa. Clin Cancer Res 2002;8:419-27.

11 Gu YZ, Schonbrunn A. Coupling specificity between somatostatin receptor sst2A and $G$ proteins: isolation of the receptor- $G$ protein complex with a receptor antibody. Mol Endocrinol 1997;11:527-37.

12 Reubi JC, Kappeler A, Waser B, et al. Immunohistochemical localization of somatostatin receptors sst2A in human tumors. Am J Pathol 1998; 153:233-45.

13 Sjolund K, Sanden G, Hakanson R, et al. Endocrine cells in human intestine: an immunocytochemical study. Gastroenterology 1983;85:1120-30

14 Moyana TN, Satkunam N. A comparative immunohistochemical study of jejunoileal and appendiceal carcinoids. Implications for histogenesis and pathogenesis. Cancer 1992;70:1081-8.

15 Dharmsathaphorn K, Racusen L, Dobbins JW. Effect of somatostatin on ion transport in the rat colon. J Clin Invest 1980;66:813-20.
16 Brand SJ, Stone D. Reciprocal regulation of antral gastrin and somatostatin gene expression by omeprazole-induced achlorhydria. J Clin Invest 1988;82:1059-66.

17 Prinz C, Sachs G, Walsh JH, et al. The somatostatin receptor subtype on rat enterochromaffinlike cells. Gastroenterology 1994;107:1067-74.

18 Aurang K, Wang J, Lloyd KC. Somatostatin inhibition of acid and histamine release by activation of somatostatin receptor subtype 2 receptors in rats. $J$ Pharmacol Exp Ther 1997;281:245-52.

19 Komasaka M, Horie S, Watanabe K, et al. Antisecretory effect of somatostatin on gastric acid via inhibition of histamine release in isolated mouse stomach. Eur J Pharmacol 2002;452:235-43.

20 Allen JP, Canty AJ, Schulz S, et al. Identification of cells expressing somatostatin receptor 2 in the gastrointestinal tract of Sstr2 knockout/lacZ knockin mice. J Comp Neurol 2002;454:329-40.

21 Piqueras L, Tache Y, Martinez V. Somatostatin receptor type 2 mediates bombesin-induced inhibition of gastric acid secretion in mice. J Physiol 2003;549:889-901

22 Bloom SR, Mortimer $\mathrm{CH}$, Thorner MO, et al. Inhibition of gastrin and gastricacid secretion by growth-hormone release-inhibiting hormone. Lancet 1974;2:1106-9.

23 Larsson LI, Goltermann N, de Magistris L, et al. Somatostatin cell processes as pathways for paracrine secretion. Science 1979;205:1393-5.

24 Kleuss C, Scherubl H, Hescheler J, et al. Selectivity in signal transduction determined by gamma subunits of heterotrimeric $G$ proteins. Science 1993;259:832-4

25 Tsutomu C, Tadataka Y. Gut somatostatin. In: Walsh JH, Graham JD, eds. Gut peptides: biochemistry and physiology. New York: Raven Press, 1994:123-45.

26 Tulassay Z. Somatostatin and the gastrointestinal tract. Scand J Gastroenterol Suppl 1998;228:115-21.

27 Hope N, Butt G, Ross I, et al. Somatostatin enhances CAMP-dependent shortcircuit current in human colon via somatostatin receptor subtype-2. Dig Dis Sci 2001:46:2499-503.

28 Nilsson 0 . Gastrointestinal carcinoids - aspects of diagnosis and classification. Apmis 1996; 104:481-92.

29 Sherman SP, Li CY, Carney JA. Microproliferation of enterochromaffin cells and the origin of carcinoid tumors of the ileum: a light microscopic and immunocytochemical study. Arch Pathol Lab Med 1979; 103:639-41.

30 Aubock L, Hofler $\mathrm{H}$. Extraepithelial intraneural endocrine cells as startingpoints for gastrointestinal carcinoids. Virchows Arch A Pathol Anat Histopathol 1983;401:17-33.

31 Lundqvist M, Wilander E. Subepithelial neuroendocrine cells and carcinoid tumours of the human small intestine and appendix. A comparative immunohistochemical study with regard to serotonin, neuron-specific enolase and S-100 protein reactivity. J Pathol 1986;148:141-7.

32 Dayal Y, DeLellis RA, Wolfe HJ. Hyperplastic lesions of the gastrointestinal endocrine cells. Am J Surg Pathol 1987;1:87-101.

33 Krenning EP, Kwekkeboom DJ, Pauwels S, et al. Somatostatin receptor scintigraphy. In: Freeman LM, eds. Nuclear medicine annual 1995. New York: Raven Press, 1995:1-50

34 Kulke MH, Mayer RJ. Carcinoid tumors. N Engl J Med 1999;340:858-68.

35 Gibril F, Venzon DJ, Ojeaburu JV, et al. Prospective study of the natural history of gastrinoma in patients with MEN1: definition of an aggressive and a nonaggressive form. J Clin Endocrinol Metab 2001;86:5282-93.

\section{EDITOR'S QUIZ: GI SNAPSHOT}

\section{Answer}

From question on page 1430

Colonoscopy showed ulcerated polypoid lesions vulnerable to contact bleeding that appeared from the transverse colon up to the terminal ileum. Histological examination was remarkable for accumulation of macrophages containing acid fast bacilli consistent with an infection of non-tuberculous mycobacteria (NTM). Mycobacterial cultures revealed growth of Mycobacterium genavense (MG). MG is a newly recognised NTM that is found mainly in patients with acquired immunodeficiency syndrome and regularly affects the digestive tract. Intestinal infections due to NTM are known to mimic Whipple's disease endoscopically and histologically. Ulcerative lesions affecting the large bowel and causing gastrointestinal bleeding represent a rare endoscopic finding.

Mycobacterial infections are uncommon complications in patients with hyper-IgE syndrome and treatment experiences are limited for this NTM species. Treatment was begun with a multidrug regimen of rifabutin, ethambutol, clarithromycin, and ciproflocazin. Three month follow up revealed regression of duodenal defects and normal mucosa on colonoscopy, indicating, to date, an adequate response to therapy. 